\title{
e-Migrinter
}

16 | 2017

Récits d'exilés

\section{Capacités de mobilité, ancrage et rapport à l'espace des jeunes des classes populaires rurales}

Séminaire d'équipe Migrinter «Théories, sources et méthodes »

Jordan Pinel et Oriana Philippe

\section{OpenEdition}

\section{Journals}

\section{Édition électronique}

URL : https://journals.openedition.org/e-migrinter/959

DOI : 10.4000/e-migrinter.959

ISSN : 1961-9685

\section{Éditeur}

UMR 7301 - Migrinter

\section{Référence électronique}

Jordan Pinel et Oriana Philippe, « Capacités de mobilité, ancrage et rapport à l'espace des jeunes des classes populaires rurales », e-Migrinter [En ligne], 16 | 2017, mis en ligne le , consulté le 20 mai 2021. URL : http://journals.openedition.org/e-migrinter/959; DOI : https://doi.org/10.4000/e-migrinter.959

Ce document a été généré automatiquement le 20 mai 2021.

Tous droits réservés 


\title{
Capacités de mobilité, ancrage et rapport à l'espace des jeunes des classes populaires rurales
}

Séminaire d'équipe Migrinter « Théories, sources et méthodes »

\author{
Jordan Pinel et Oriana Philippe
}

\section{RÉFÉRENCE}

«Capacités de mobilité, ancrage et rapport à l'espace des jeunes des classes populaires rurales ", Séminaire d'équipe Migrinter « Théories, sources et méthodes », scéance par Thomas Venet, le 19 janvier, Poitiers.

1 Le séminaire "Théories, sources et méthodes" a fait sa rentrée au laboratoire Migrinter le 19 janvier 2017, sous l'impulsion de Naïk Miret, Celio Sierra-Paycha et Marie-Françoise Valette. Outre le fait de réunir l'équipe une fois par mois, ce rendezvous important de la vie du laboratoire permet d'élargir les réflexions relatives aux théories des migrations. Lors de cette première séance de reprise, Thomas Venet est venu présenter les résultats de sa thèse en sociologie et démographie qu'il a soutenue le 24 novembre 2016 (Université de Picardie Jules Verne), sous la direction de Virginie De Luca Barrusse (Centre de Recherches de l'Institut de Démographie de l'Université Paris 1) et de Catherine Bonvalet (Institut national d'études démographiques). Intitulée « La jeunesse populaire en territoires désindustrialisés. Famille, emploi et sociabilité en contexte rural » (Venet, 2016), sa thèse interroge la capacité de mobilité et les rapports à l'espace des jeunes issus des classes populaires rurales de Picardie. C'est donc un peu plus de deux mois après sa soutenance qu'il est venu au laboratoire Migrinter, évoquer son travail de thèse et nous faire part de quelques-uns de ses résultats. Son intervention a été discutée par Sarah Przybyl, dont la thèse intitulée « Territoires de la migration, territoires de la protection. Parcours et expériences des mineurs isolés 
étrangers accueillis en France» (Przybyl, 2016) a quant à elle été soutenue le 12 décembre 2016 (Université de Poitiers).

2 Thomas Venet a débuté son propos en resituant le cadre de sa recherche. Il s'est ainsi intéressé à la jeunesse populaire, et notamment aux modalités de passage à «l'âge adulte ». Ce passage est analysé par le sociologue comme " un ensemble de processus et stratégies issus de la socialisation et prises dans des mondes socio-spatialisés ». Il s'est plus particulièrement intéressé à ces modalités de passage à l'âge adulte eu égard au processus d'autonomisation et d'accès aux ressources financières de ces jeunes. Considérant que les dispositions sociales et les visions de l'avenir de ces jeunes qui cherchent à accéder à l'autonomie sont «issues de la socialisation de classe et de genre » dans des contextes locaux spécifiques, Thomas Venet a indiqué avoir choisi d'éviter une trop large comparaison. Il a donc ancré sa recherche dans des environnements locaux, faisant le choix d'un territoire rural où l'industrie faisait vivre une grande partie de la population jusque dans les années 1970. Il a sélectionné et mobilisé deux territoires picards, parmi un ensemble de cantons préalablement classifiés comme des territoires désindustrialisés ou en voie de désindustrialisation, marqués par de forts taux de chômage, un fort dépeuplement et un vieillissement de la population. Ces territoires présentent un intérêt certain quant à l'étude des modalités d'accès aux ressources financières et d'autonomisation de ces jeunes. Les rencontres avec les enquêtés ont notamment commencé par le biais des institutions d'insertion professionnelle et se sont ensuite poursuivies en dehors de celles-ci, évitant ainsi au chercheur d'être assimilé à ces structures par les enquêtés.

Reprenant une démarche adoptée par Nicole Tabard dans les années 1990, Thomas Venet a questionné la «dimension spatiale » de ces mondes sociaux. Il a ainsi abordé le rapport à l'espace et à la mobilité de ces jeunes au moment du passage à l'âge adulte ${ }^{1}$ et leur capacité à accéder aux ressources financières et à l'autonomie. Au cours de sa recherche, la mobilité va se révéler comme un élément déterminant et même parfois comme un levier. Ainsi, Thomas Venet réalise une première distinction entre les jeunes qui vont devenir ceux qu'il nomme les "gars du coin", par opposition à ceux qui parviennent à réaliser un processus de mobilité ou de " décohabitation partielle » afin d'aller étudier ailleurs, devenant alors «ceux du weekend ». Cette première mobilité est déterminée par plusieurs éléments et notamment la possibilité d'accéder à des ressources matérielles (par le biais d'aides familiales par exemple) afin de financer un logement ou les études, mais également par la capacité à se " projeter ailleurs » et à se "construire de nouvelles identités sociales", lesquelles nécessitent de pouvoir se couper du contexte local.

4 Ensuite, le sociologue met en lumière une seconde distinction déterminée également par le facteur de mobilité, mais cette fois parmi les jeunes restés dans la région. Au cours de sa recherche, il a en effet réalisé combien le fait d'avoir le permis de conduire et d'être détenteur d'un véhicule donnent aux jeunes une capacité de flexibilité qui leur permet d'intégrer plus facilement le marché de l'emploi. Dans une région où réside un très fort taux de chômage et où l'écart entre les demandeurs d'emploi et les offres est très important, cet élément se révèle finalement très significatif. Si les emplois obtenus ne sont pas toujours stables et fixes, la capacité à obtenir des emplois à durée déterminée, et notamment d'effectuer des remplacements ponctuels, va cependant permettre à certains jeunes d'obtenir des ressources financières et une expérience, indispensables dans leur processus d'autonomisation. A contrario, le fait de ne pas (ou 
plus) avoir le permis de conduire ou celui de ne pas être véhiculé, va être directement ressenti par les jeunes comme un empêchement à l'insertion professionnelle. Ceux qui en sont dépourvus vont généralement considérer que leur situation ne pourra pas évoluer tant qu'ils n'auront pas acquis ces instruments de mobilité. Ici encore, le bénéfice de cette mobilité est conditionné à un accès aux ressources matérielles, la distinction s'opérant notamment entre les jeunes bénéficiant d'aides familiales pour payer le permis et financer un véhicule et ceux n'en ayant pas la possibilité.

Dans ces deux cas, la mobilité joue un rôle essentiel dans le processus d'autonomisation et d'accès aux ressources, par le fait de changer de lieu de vie ou de devenir un travailleur mobile.

6 Thomas Venet poursuit cette observation en soulignant qu'à travers le ressenti de ces jeunes enquêtés, la mobilité se présente aussi comme un outil pour accéder à l'autonomie. Elle est d'ailleurs envisagée par eux comme une option et une solution dès lors qu'elle est susceptible d'offrir un emploi. Elle ne s'inscrit alors pas comme un souhait ou une volonté mais comme une nécessité, une porte d'accès au monde professionnel. Ainsi, une partie des jeunes déclarent être prêts à se résoudre à aller vivre ailleurs, y compris dans des villes qui leur déplaisent, pourvu que l'incertitude d'un départ soit contrebalancée par la possibilité d'un emploi «qui vaille le coût ». De la même façon, plusieurs jeunes envisagent les métiers de l'armée qui les contraindraient certes à une mobilité mais leur offriraient la garantie d'un emploi stable, d'un recrutement plus simple, de nouveaux débouchés, d'une carrière, d'une retraite, voire d'une possible ascension sociale. Dans ce cadre, même s'ils doivent rompre avec leur contexte local sans réelle envie, ces jeunes acceptent cette mobilité géographique encadrée par ce parcours professionnel.

7 Pour conclure cette intervention, Thomas Venet est revenu sur la relation paradoxale qu'il a observée pendant sa recherche entre les jeunes et l'espace. La mobilité se présente pour ces enquêtés comme un désir d'ailleurs se construisant sur la volonté d'échapper à une réalité sociale qui les enserre. Ceci se traduit notamment par un fort rejet de leur lieu de vie, constaté de façon presque unanime au cours de l'enquête sur les deux terrains, et lu par Thomas Venet comme une « lutte de soi contre soi » (Lahire, 2005). Le sociologue explique ce rejet par la réappropriation par les jeunes des stigmates dont font l'objet les mondes ruraux et populaires dans les médias locaux et nationaux - populations assimilées au chômage, à la pauvreté et qualifiées de «villages de cas soc' ", "chômeurs-land», en opposition aux classes dominantes urbaines - et dont ils souhaitent se distancer en invoquant cette volonté de partir. Ce souhait d'échapper à ce lieu de vie déprécié est cependant ambivalent car généralement pas ou peu de projets de départ concrets émergent. Ainsi, de manière paradoxale, la recherche d'emploi se fait généralement dans une zone géographique restreinte autour du lieu de vie. Le sociologue constate que ceci ne s'explique pas seulement par la difficulté matérielle à être mobile. Sa recherche met aussi en exergue l'importance des réseaux d'interconnaissances et d'entraides locales pour ces jeunes, lesquels instituent des systèmes de solidarité, de relations et de circulation de l'information. Pour ces jeunes, ces réseaux constituent un cadre sécurisant et rassurant, un « rempart ultime contre les crises d'appartenances professionnelles et familiales " (Renahy, 2010). Pourtant, si ces réseaux pourvoient d'un côté à la reconnaissance sociale de ces jeunes, il est vrai également qu'ils les «figent » dans ce contexte local. D'ailleurs il est symptomatique que plusieurs jeunes envisagent une mobilité pour des raisons professionnelles, mais 
indiquent malgré tout qu'il leur serait indispensable de revenir régulièrement. Une affirmation qui semble paradoxale face au rejet du lieu d'origine.

Si au sein du laboratoire Migrinter, nous sommes davantage habitués à aborder la mobilité à une échelle internationale, cette recherche présente l'intérêt de mettre en lumière l'importance du rapport à l'espace et à la mobilité dans un contexte local. D'ailleurs, ce rejet d'un lieu de provenance en difficulté économique et la volonté de partir ou encore l'importance d'être mobile pour accéder à un emploi ou des études sont des phénomènes que l'on peut observer également dans le cadre des migrations internationales.

Lors de la discussion, Sarah Przybyl a d'ailleurs évoqué plusieurs parallèles entre les observations de Thomas Venet et sa propre recherche auprès des mineurs isolés étrangers. Elle note que ces jeunes, aux parcours migratoires et de mobilité divers, rapportent eux aussi un sentiment de «blocage " au moment de leur placement dans des foyers de l'enfance situés dans des communes rurales. Pour les jeunes étrangers, la mobilité représente " la seule issue pour réussir ». Elle constate également que dans les deux cas, un « capital économique, spatial et social » est nécessaire à ces mobilités. Les différents symboles des sociétés de consommation alimentent l'image d'un milieu rural qui enferme et contraint à l'immobilité. Ce constat dressé par la géographe pourrait être appliqué aussi bien dans ce contexte picard que dans celui des migrations internationales. On constate à partir des résultats de ces deux thèses que travailler sur le rapport à l'espace de ces jeunes requiert un dialogue entre sociologie et géographie. C'était notamment l'une des remarques de Sarah Przybyl qui confirme une nouvelle fois la frontière « poreuse » entre ces deux disciplines.

\section{BIBLIOGRAPHIE}

Lahire, Bernard (2005) Distinctions culturelles et lutte de soi contre soi : « détester la part populaire de soi », Hermès, La Revue, n 42, pp. 137-143.

Przybyl, Sarah (2016) Territoires de la migration, territoires de la protection. Parcours et expériences des mineurs isolés étrangers accueillis en France, Poitiers, Université de Poitiers, 501 p.

Th. Doct. : Géogr. : Poitiers : 2016.

Renahy, Nicolas (2010) Les gars du coin. Enquête sur une jeunesse rurale, Paris, La Découverte, 285 p.

Venet, Thomas (2016) La jeunesse populaire en territoires désindustrialisés. Famille, emploi et sociabilité en contexte rural, Amiens, Université de Picardie Jules Verne, 305 p.

Th. Doct. : Sociologie et Démographie : Amiens : 2016. 


\section{NOTES}

1. Le passage à l'âge adulte est «entendu comme période d'accroissement d'une autonomie relative vis-à-vis de la famille d'origine, en accédant à des statuts sociaux d'adulte et à des moyens de subsistances propres » selon Thomas Venet.

INDEX

Index géographique : France

Mots-clés : jeunes, mobilité spatiale, intégration sociale, étude régionale

\section{AUTEURS}

JORDAN PINEL

Doctorant en géographie, Migrinter - UMR 7301, CNRS / Université de Poitiers jordan.pinel@gmail.com

\section{ORIANA PHILIPPE}

Doctorante en géographie, Migrinter - UMR 7301, CNRS / Université de Poitiers oriana.philippe@univ-poitiers.fr 